Supporting information

\title{
Hydrogen Sensing at Room Temperature Using Flame- synthesized Palladium-decorated Crumpled Reduced Graphene Oxide Nanocomposites
}

Mohammad Moein Mohammadi, Abhishek Kumar, Jun Liu, Yang Liu, Thomas Thundat, Mark T. Swihart

Department of Chemical and Biological Engineering, University at Buffalo, The State University of New York, Buffalo, NY 14260, USA 


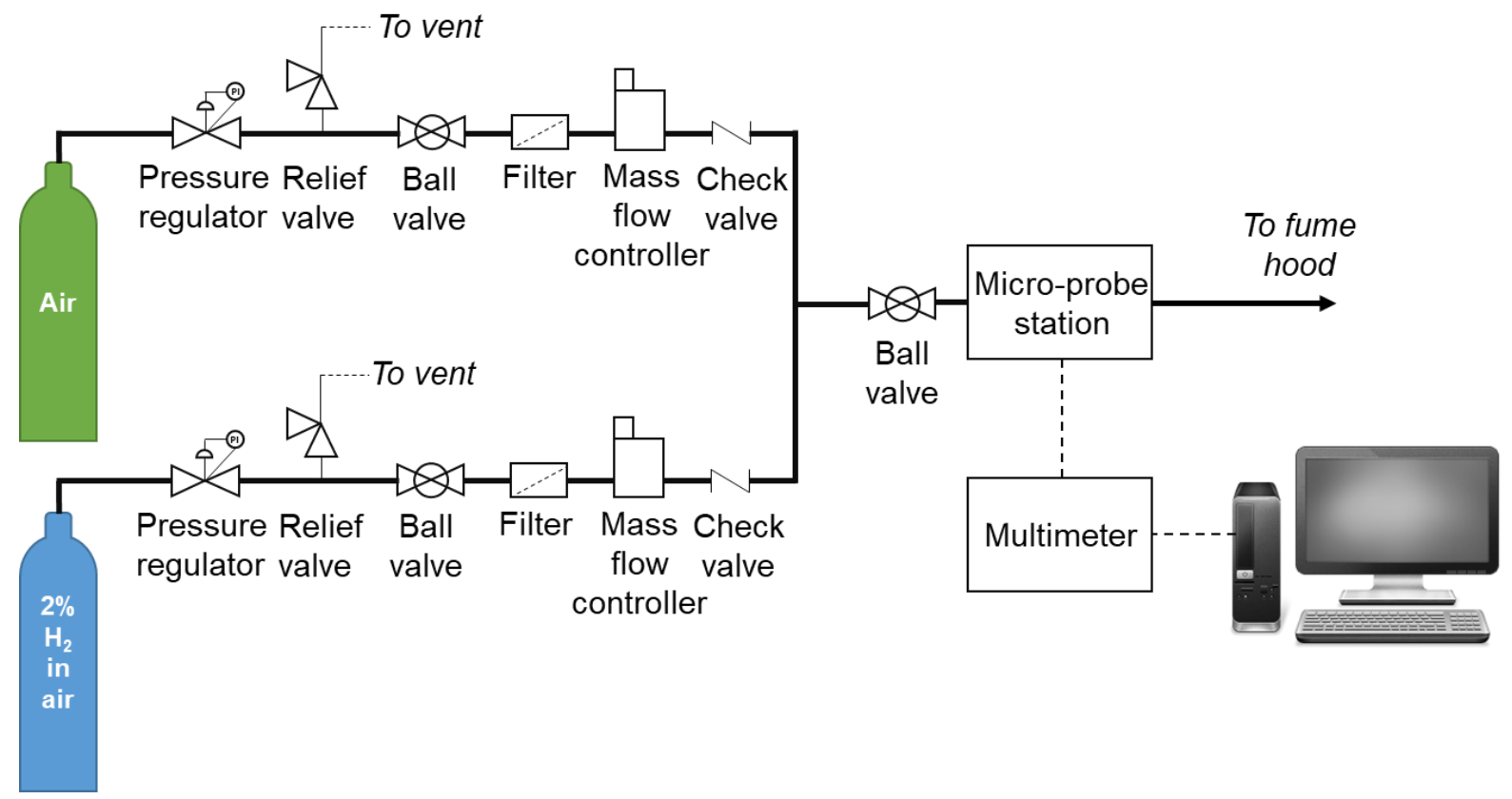

Figure S1. Schematic of the sensor testing apparatus. 

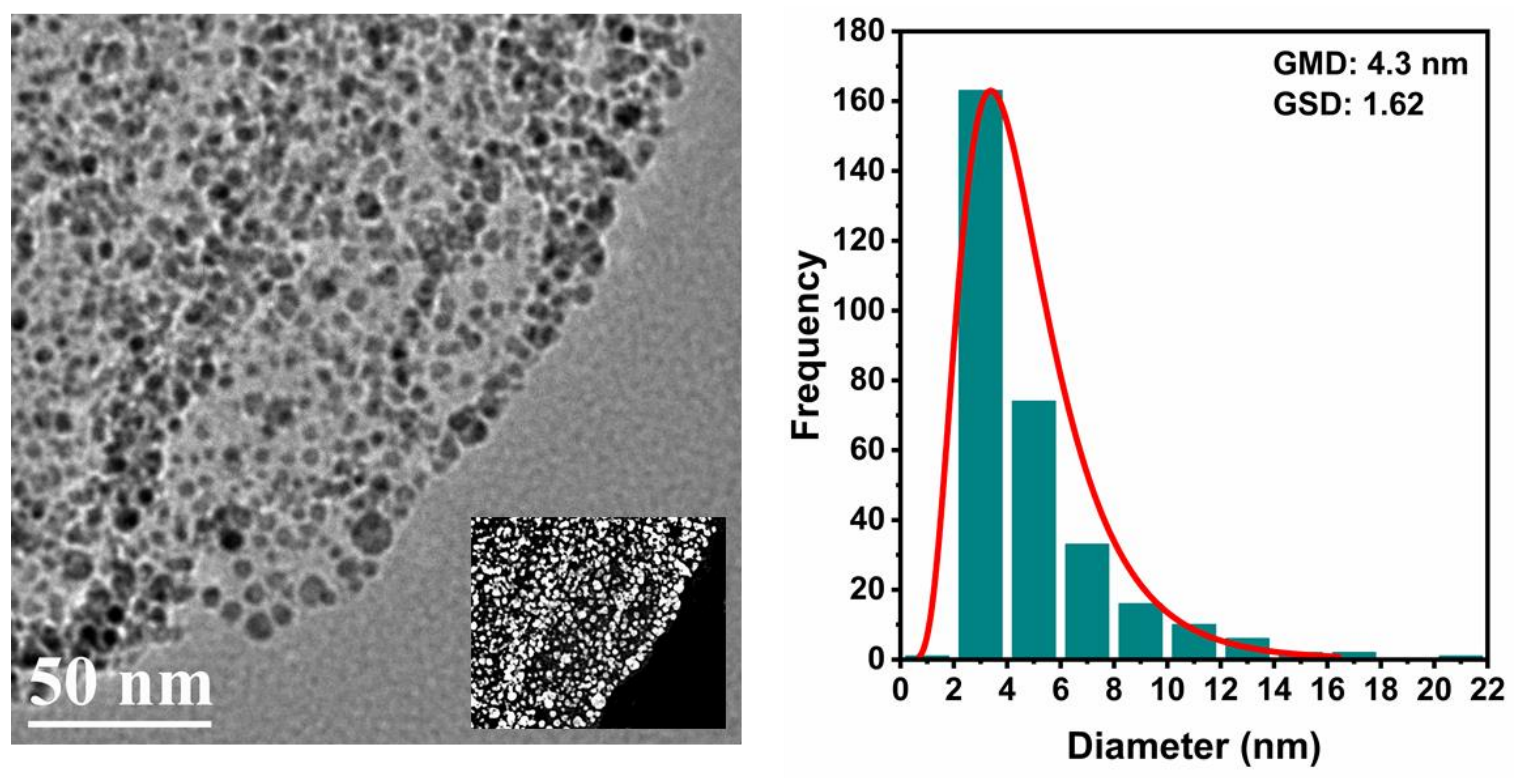

Figure S2. TEM image and the corresponding particle size distribution for PdCGB. 


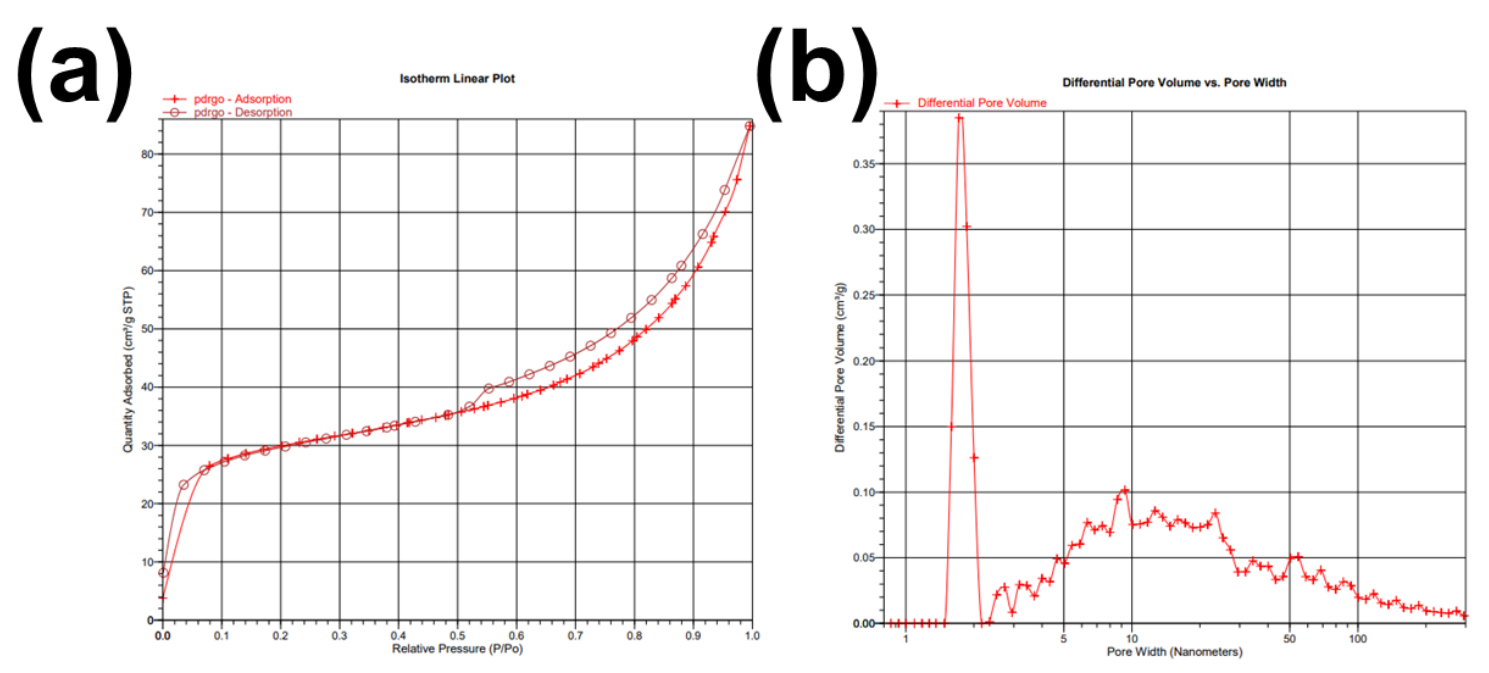

Figure S3. a) Nitrogen physisorption isotherm used for BET surface area analysis of Pd-CGB; b) differential pore volume versus pore width of Pd-CGB based on the BJH analysis. 


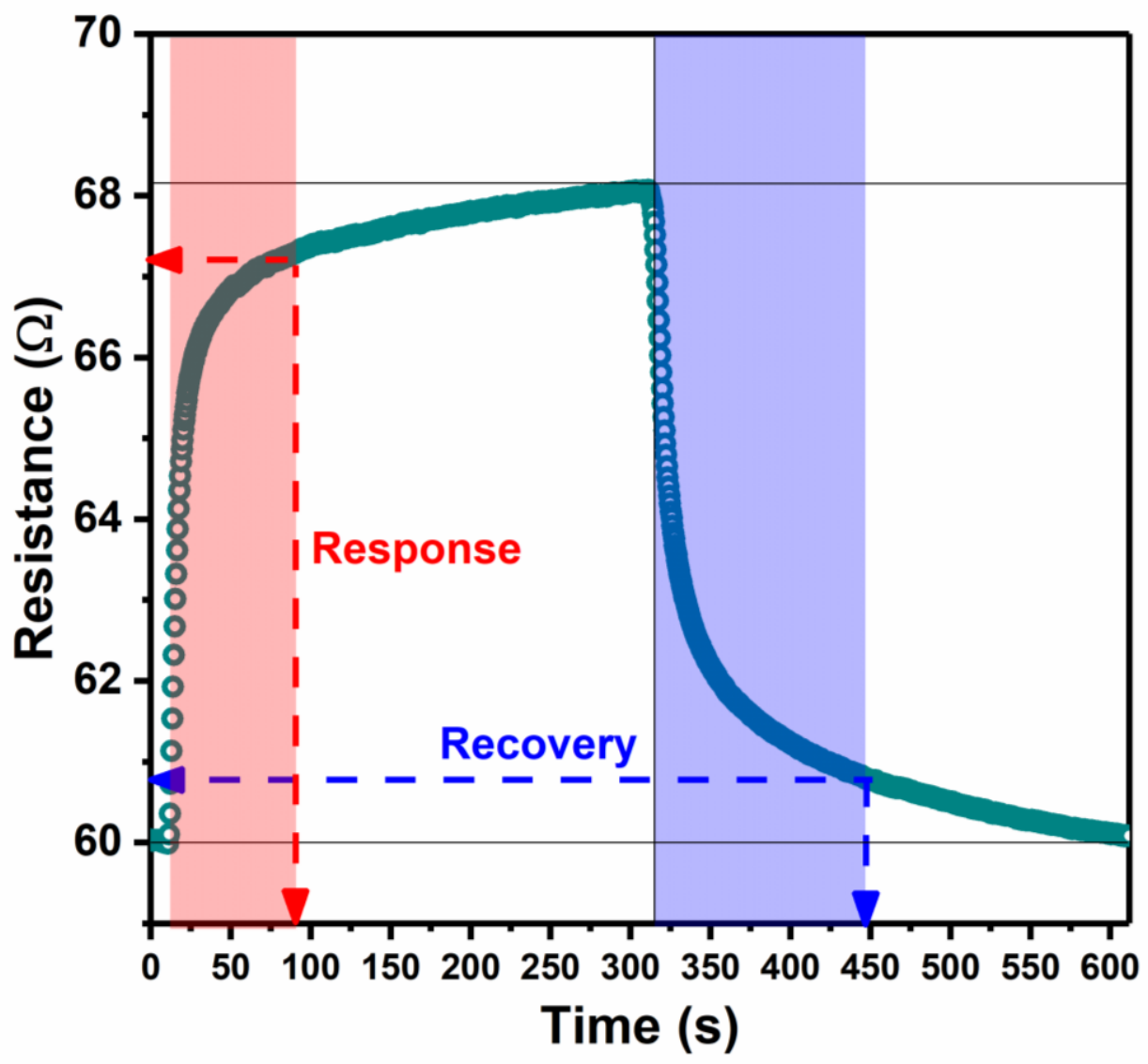

Figure S4. Detailed response and recovery time extraction based on $90 \%$ change in resistance for $2 \% \mathrm{H}_{2}$ response. 


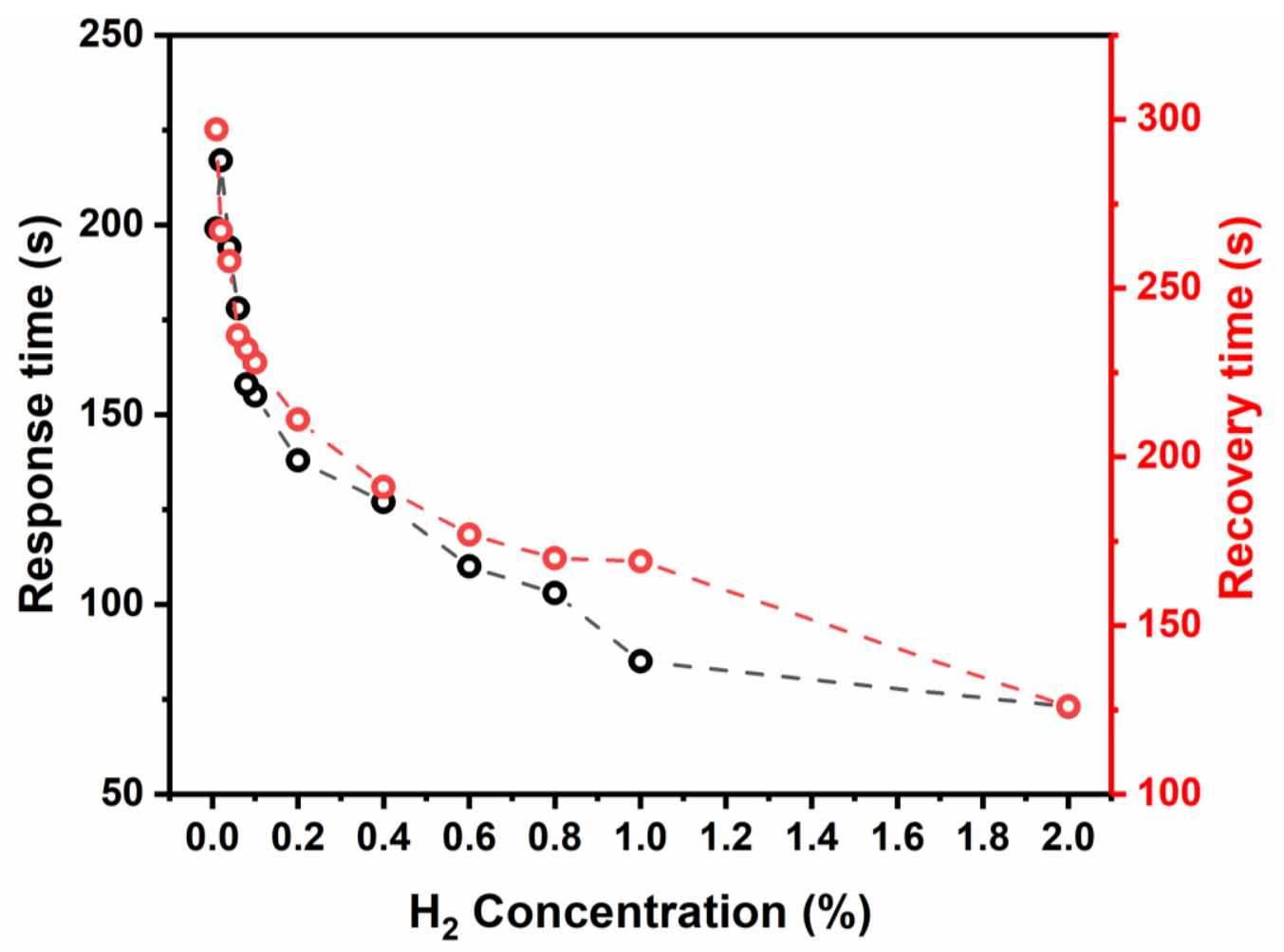

Figure S5. Response and recovery times for various $\mathrm{H}_{2}$ concentrations from 0.01 to $2 \%$. 
Table S1. Pd-graphene chemo-resistive sensors for $\mathrm{H}_{2}$ detection in air at room temperature

\begin{tabular}{|c|c|c|c|c|c|c|c|c|c|}
\hline No. & $\begin{array}{l}\text { Sensing } \\
\text { material }\end{array}$ & Synthesis method & $\begin{array}{l}\text { Response } \\
\text { value }\end{array}$ & $\begin{array}{l}\text { Response } \\
\text { time }\end{array}$ & $\begin{array}{l}\text { Recovery } \\
\text { time }\end{array}$ & $\begin{array}{l}\text { Sensing } \\
\text { range (\%) }\end{array}$ & $\begin{array}{l}\text { LOD } \\
\text { (ppm) }\end{array}$ & Year & Ref. \\
\hline 1 & Pd-CGB & $\begin{array}{l}\text { Reduction of Pd and } \\
\text { GO using Flame }\end{array}$ & $14.8 \%$ at $2 \%$ & $73 \mathrm{~s}$ & $126 \mathrm{~s}$ & $0.0025-2$ & 25 & 2020 & $\begin{array}{l}\text { This } \\
\text { report }\end{array}$ \\
\hline 2 & $\mathrm{Pd} N \mathrm{~N} / \mathrm{G}$ & $\begin{array}{c}\text { Chemical decoration - } \\
\text { CVD }\end{array}$ & $5.9 \%$ at $1 \%$ & $180 \mathrm{~s}$ & $540 \mathrm{~s}$ & $1-4$ & 10000 & 2019 & [1] \\
\hline 3 & $\begin{array}{l}\text { 3D porous laser- } \\
\text { induced graphene } \\
\text { (LIG) decorated } \\
\text { with } \mathrm{Pd}\end{array}$ & $\begin{array}{l}\text { e-beam evaporation - } \\
\text { Laser on polyimide }\end{array}$ & $3.3 \%$ at $1 \%$ & NA & NA & $0.06-1.3$ & 600 & 2019 & [2] \\
\hline 4 & $\begin{array}{l}\text { Pd-functionalized, } \\
\text { suspended } \\
\text { graphene }\end{array}$ & $\begin{array}{c}\text { e-beam deposition - } \\
\text { CVD }\end{array}$ & $\begin{array}{l}1.5 \% \text { at } \\
0.01 \%\end{array}$ & $42 \mathrm{~s}^{1}$ & NA & $0.001-0.01$ & 10 & 2018 & [3] \\
\hline 5 & $\mathrm{Pd} N \mathrm{~N} / \mathrm{G}$ & $\begin{array}{l}\text { Chemical reduction of } \\
\text { Pd - Liquid Phase } \\
\text { Exfoliation of graphite }\end{array}$ & $26 \%$ at $1 \%$ & $40 s^{2}$ & $490 \mathrm{~s}^{3}$ & 1 & 10000 & 2017 & [4] \\
\hline 6 & $\mathrm{Pt}-\mathrm{Pd} / \mathrm{rGO}$ & $\begin{array}{l}\text { Chemical reduction of } \\
\text { Pd and GO }\end{array}$ & $52 \%$ at $0.8 \%$ & $\sim 720 \mathrm{~s}$ & $\sim 600 \mathrm{~s}$ & $0.005-0.8$ & 50 & 2016 & {$[5]$} \\
\hline 7 & $\begin{array}{l}\text { Nanoporous Pd- } \\
\text { rGO }\end{array}$ & $\begin{array}{c}\text { Chemical reduction of } \\
\mathrm{Pd} \text { and GO }\end{array}$ & $30.6 \%$ at $1 \%$ & $360 \mathrm{~s}$ & $120 \mathrm{~s}$ & $0.00001-1$ & 1 & 2015 & {$[6]$} \\
\hline 8 & $\mathrm{Pd} \mathrm{NP} / \mathrm{rGO}$ & Microwave - CVD & $14.7 \%$ at $1 \%$ & $\sim 30 \mathrm{~s}$ & $450 \mathrm{~s}$ & $0.01-5$ & 100 & 2015 & [7] \\
\hline 9 & $\mathrm{Pd} N \mathrm{~N} / \mathrm{G}$ & $\begin{array}{c}\text { Thermal evaporation - } \\
\text { CVD }\end{array}$ & $\begin{array}{l}6.7 \% \text { at } \\
0.0015 \%\end{array}$ & $\sim 59 \mathrm{~s}^{4}$ & $\sim 400 \mathrm{~s}$ & $0.0002-0.0015$ & 2 & 2015 & [8] \\
\hline 10 & $\begin{array}{c}\text { Pt/Pd core-shell- } \\
\text { G }\end{array}$ & $\begin{array}{c}\text { Chemical reduction of } \\
\mathrm{Pd}, \mathrm{Pt} \text { and } \mathrm{GO}\end{array}$ & $36 \%$ at $1 \%$ & $180 \mathrm{~s}$ & $72 \mathrm{~s}$ & $0.00001-4$ & 1 & 2015 & [9] \\
\hline 11 & $\begin{array}{l}\text { Pd Nanocube- } \\
\text { rGO }\end{array}$ & $\begin{array}{c}\text { Chemical reduction of } \\
\mathrm{Pd} \text { and } \mathrm{GO}\end{array}$ & $11 \%$ at $0.1 \%$ & $1080 \mathrm{~s}$ & $2400 \mathrm{~s}$ & $0.0006-0.1$ & 6 & 2014 & {$[10]$} \\
\hline 12 & NiPd-rGO & $\begin{array}{c}\text { Chemical reduction of } \\
\mathrm{Pd}, \mathrm{Ni} \text { and } \mathrm{GO}\end{array}$ & $11 \%$ at $0.1 \%$ & $180 \mathrm{~s}$ & $7200 \mathrm{~s}$ & $0.00001-1$ & 1 & 2014 & [11] \\
\hline 13 & $\begin{array}{l}\text { Pd Nanocube- } \\
\text { rGO }\end{array}$ & $\begin{array}{c}\text { Chemical reduction of } \\
\mathrm{Pd} \text { and } \mathrm{GO}\end{array}$ & $17.7 \%$ at $1 \%$ & $450 \mathrm{~s}$ & $660 \mathrm{~s}$ & $0.001-1$ & 10 & 2014 & [12] \\
\hline
\end{tabular}




\begin{tabular}{|c|c|c|c|c|c|c|c|c|c|}
\hline 14 & $\begin{array}{l}\text { palladium- } \\
\text { decorated } \\
\text { graphene } \\
\text { nanoribbon } \\
\text { film/SiO2/Si }\end{array}$ & $\begin{array}{l}\text { Chemical reduction of } \\
\mathrm{Pd} \text { and } \mathrm{GO} \text { ribbons }\end{array}$ & $2000 \%$ at $1 \%$ & $625 \mathrm{~s}$ & NA & $0.01-1$ & 100 & 2014 & [13] \\
\hline 15 & $\begin{array}{c}P d \text { film/exfoliated } \\
G\end{array}$ & $\begin{array}{l}\text { Magnetron sputtering } \\
\text { - exfoliation }\end{array}$ & $4.5 \%$ at $1 \%$ & $8 s^{4}$ & $210 s$ & $0.005-2$ & 50 & 2013 & [14] \\
\hline 16 & $\mathrm{Pd} N \mathrm{~N} / \mathrm{G}$ & $\begin{array}{l}\text { e-beam evaporation - } \\
\text { CVD }\end{array}$ & $\begin{array}{l}4.1 \% \text { at } \\
0.05 \%\end{array}$ & $213 s$ & $463 \mathrm{~s}$ & $0.0025-1$ & 25 & 2010 & [15] \\
\hline \multicolumn{10}{|c|}{$\begin{array}{l}{ }^{1} \text { defined as the time to reach half of the value } \\
{ }^{2} \text { defined as the time between } 10 \% \text { to } 90 \% \text { of the response } \\
{ }^{3} \text { defined as the time between } 90 \% \text { to } 10 \text { of the recovery } \\
{ }^{4} \text { defined as the time to reach }\left(e^{-1}=36.8 \%\right)\end{array}$} \\
\hline
\end{tabular}




\section{References}

[1] X. Tang, P.A. Haddad, N. Mager, X. Geng, N. Reckinger, S. Hermans, M. Debliquy, J.P. Raskin, Chemically deposited palladium nanoparticles on graphene for hydrogen sensor applications, Sci Rep, 9 (2019) 3653.

[2] J. Zhu, M. Cho, Y. Li, I. Cho, J.-H. Suh, D. Del Orbe, Y. Jeong, T.-L. Ren, I. Park, Biomimetic Turbinate-like Artificial Nose for Hydrogen Detection Based on 3D Porous Laser-Induced Graphene, ACS Appl. Mater. Interfaces, 11 (2019) 41.

[3] T. Yokoyama, T. Tanaka, Y. Shimokawa, R. Yamachi, Y. Saito, K. Uchida, Pd-Functionalized, Suspended Graphene Nanosheet for Fast, Low-Energy Multimolecular Sensors, ACS Applied Nano Materials, 1 (2018) 3886-3894.

[4] B. Alfano, E. Massera, T. Polichetti, M.L. Miglietta, G. Di Francia, Effect of palladium nanoparticle functionalization on the hydrogen gas sensing of graphene based chemiresistive devices, Sensors and Actuators B: Chemical, 253 (2017) 1163-1169.

[5] Y. Peng, J. Ye, L. Zheng, K. Zou, The hydrogen sensing properties of Pt-Pd/reduced graphene oxide based sensor under different operating conditions, RSC Advances, 6 (2016) 2488024888.

[6] D.-T. Phan, G.-S. Chung, A novel nanoporous Pd-graphene hybrid synthesized by a facile and rapid process for hydrogen detection, Sensors and Actuators B: Chemical, 210 (2015) 661-668.

[7] R.D. Martínez-Orozco, R. Antaño-López, V. Rodríguez-González, Hydrogen-gas sensors based on graphene functionalized palladium nanoparticles: impedance response as a valuable sensor, New Journal of Chemistry, 39 (2015) 8044-8054.

[8] J. Yi, S.H. Kim, W.W. Lee, S.S. Kwon, W.N. Sung, W.I. Park, Graphene meshes decorated with palladium nanoparticles for hydrogen detection, Journal of Physics D: Applied Physics, 48 (2015).

[9] D.-T. Phan, A.S.M.I. Uddin, G.-S. Chung, A large detectable-range, high-response and fastresponse resistivity hydrogen sensor based on $\mathrm{Pt} / \mathrm{Pd}$ core-shell hybrid with graphene, Sensors and Actuators B: Chemical, 220 (2015) 962-967.

[10] D.-T. Phan, G.-S. Chung, A novel Pd nanocube-graphene hybrid for hydrogen detection, Sensors and Actuators B: Chemical, 199 (2014) 354-360.

[11] D.T. Phan, G.S. Chung, Reliability of hydrogen sensing based on bimetallic Ni-Pd/graphene composites, International Journal of Hydrogen Energy, 39 (2014) 20294-20304.

[12] D.-T. Phan, G.-S. Chung, Effects of Pd nanocube size of Pd nanocube-graphene hybrid on hydrogen sensing properties, Sensors and Actuators B: Chemical, 204 (2014) 437-444.

[13] Z. Zhang, Q. Xue, Y. Du, C. Ling, W. Xing, Highly enhanced sensitivity of hydrogen sensors using novel palladium-decorated graphene nanoribbon film/SiO2/Si structures, J. Mater. Chem. A, 2 (2014) 15931-15937.

[14] J. Lim, S. Hwang, H.S. Yoon, E. Lee, W. Lee, S.C. Jun, Asymmetric electron hole distribution in single-layer graphene for use in hydrogen gas detection, Carbon, 63 (2013) 3-8.

[15] W. Wu, Z. Liu, L.A. Jauregui, Q. Yu, R. Pillai, H. Cao, J. Bao, Y.P. Chen, S.-S. Pei, Waferscale synthesis of graphene by chemical vapor deposition and its application in hydrogen sensing, Sensors and Actuators B: Chemical, 150 (2010) 296-300. 